\title{
Pilot Study on Continuous Hyperfractionated Accelerated Radiotherapy (CHART) and High Dose Rate Brachytherapy in Locally Advanced Cervical
} Cancer

\author{
Biswa Mohan Biswal ${ }^{1 *}$, Nik Min Ahmad ${ }^{1}$, Zainal
}

${ }^{3}$, Ahmad Zakaria ${ }^{1}$ and Nor Hayati Othman ${ }^{2}$

${ }^{1}$ Department of Nuclear medicine, Radiotherapy and Oncology, School of Medical Sciences, Universiti Sains Malaysia, Kubang Kerian, Kelantan, Malaysia ${ }^{2}$ Department of Pathology, School of Medical Sciences, Universiti Sains Malaysia, Kubang Kerian, Kelantan, Malaysia

${ }^{3}$ Department of Obstretics and Gynecology, Hospital Raja Perempuan Zainab-Il, Kota Bharu, Kelantan, Malaysia

\begin{abstract}
Background: Fractionated external beam radiotherapy followed by intracavitary brachytherapy is the mainstay of treatment in cervical cancer. Due to long radiotherapy course, patients in developing countries default effective radiotherapy. Treatment break or discontinuance leads to treatment failure. Continuous hyperfractionated accelerated radiotherapy (CHART) is an altered fractionation scheme used to reduce overall treatment time, overcome tumor repopulation thus could be an option in cervical carcinoma.
\end{abstract}

Methods: Twenty-five histopathological documented locally advanced cervical carcinoma (FIGO stage IB2IVA) were treated with $42 \mathrm{~Gy} / 30$ fractions external radiotherapy over 10-day period at an inter-fraction interval of 6 hours using CHART technique. Following CHART, patients were subjected to high dose rate brachytherapy to a dose schedule of $9 \mathrm{Gyx} 2$ at weekly interval. The response to above radiotherapy protocol and morbidity pattern was evaluated.

Results: All patients completed planned CHART external radiotherapy. The median follow-up was 32-months with a pelvic control rate of $68 \%$ and 24 -month overall survival was $72 \%$. The most common acute toxicity in CHART technique was fatigue $(64 \%)$ and diarrhea $(52 \%)$, the late effects being rectal bleeding (2-cases), subcutaneous fibrosis (1-case), spinal pain (1-case).

Conclusion: CHART technique is an effective and well tolerated technique in locally advanced cervical cancer at a cost of manageable fatigue and diarrhea. Further investigation involving large number of patients is warranted to evaluate this technique.

Keywords: Cervical cancer; Radiotherapy; CHART; HDR brachytherapy; Morbidity; Response rate

\section{Introduction}

Management of locally advanced cervical cancer is a challenge to oncologist. The mainstay of treatment in cancer cervix is radiotherapy. A desired proportion of external beam radiation and intracavitary brachytherapy is most commonly followed worldwide. External beam radiotherapy delivers uniform irradiation to a block of tissue including tumor volume and safe margins in the pelvis. The standard external radiotherapy consists of a dose per fraction of 1.8-2.25 Gy per day treating five-days-a-week for 4-5 weeks with 2-days weekend break. Following external beam radiotherapy, intracavitary brachytherapy is supplemented to increase radiation dose to the primary tumor. Currently recommendation of brachytherapy is in transition: shifting from classical low dose rate (LDR) system to multifractionated high dose rate (HDR) system [1]. Multiple randomized clinical trials have documented equi-effectiveness of HDR in place of classical low dose rate brachytherapy. The right fractionation scheme is still debatable however a fractionation of 2-3 fractions seem more practical in clinical practice [2].Though concurrent chemo-radiotherapy is the standard of care in many developed countries [3,4], the above technique in bulky disease has been questioned by some authors due to prohibitive toxicity and discontinuation of radiotherapy in developing world $[5,6]$. In developing countries, long treatment course and travel to radiotherapy centers every day resulted in discontinuance of effective radiotherapy treatment.

Radiobiological challenges are of paramount importance to decide about fractionation to limit late morbidity and increased dose to improve local control rate. Altered fractionation schemes like hyperfractionation or hypofractionation and its modifications have been tried to improve radiation dose and decrease overall treatment time [7]. Further, repopulation of clonogens during radiotherapy might result in radioresistance. Breaks during radiation therapy are known to decrease local control rates [8]. Saunders and their coworkers from Mount Vernon Hospital London have popularized continuous hyperfractionated accelerated radiotherapy (CHART) technique to reduce dose per fraction, total tumor dose, and decrease overall treatment time, so that the treatment schedule finish before development of radiation morbidity and repopulation of clonogens. CHART radiotherapy scheme have shown to be effective in head \& neck, lung, and rectal cancers with tolerable acute and late morbidity

*Corresponding author: Dr. Biswa Mohan Biswal. MBBS.MD.DNB, Associate Professor, Radiotherapy \& Oncology, Department of Nuclear Medicine, Radiotherapy \& Oncology, School of Medical Sciences, Health Campus, Universiti Sains Malaysia, 16150 Kubang Kerian, Kelantan, Malaysia, Tel: +60-9-7676666; Fax: +60-97653370; E-mail: biswa@kb.usm.my

Received March 29, 2011; Accepted July 20, 2011; Published July 22, 2011

Citation: Biswal BM, Ahmad NM, Hanafia ZA, Zakaria A, Othman NH, et al. (2011) Pilot Study on Continuous Hyperfractionated Accelerated Radiotherapy (CHART) and High Dose Rate Brachytherapy in Locally Advanced Cervical Cancer. J Cancer Sci Ther 3: 125-129. doi:10.4172/1948-5956.1000073

Copyright: (C) 2011 Biswal BM, et al. This is an open-access article distributed under the terms of the Creative Commons Attribution License, which permits unrestricted use, distribution, and reproduction in any medium, provided the original author and source are credited. 
Citation: Biswal BM, Ahmad NM, Hanafia ZA, Zakaria A, Othman NH, et al. (2011) Pilot Study on Continuous Hyperfractionated Accelerated Radiotherapy (CHART) and High Dose Rate Brachytherapy in Locally Advanced Cervical Cancer. J Cancer Sci Ther 3: 125-129. doi:10.4172/1948-5956.1000073

$[7,9,10]$. Here we would like to try 10 -days CHART radiotherapy technique for the management of locally-advanced cervical cancer.

\section{Materials and Methods}

This is a pilot study using external beam 10-day CHART technique for locally advanced cervical cancer. In addition, patients received two fractions of high dose rate brachytherapy within 1-2 weeks following external radiotherapy. The patients were pooled from the oncology clinics of Radiotherapy unit of University hospital, Universiti Sains Malaysia from 2003-2007. This research was reviewed by the research \& ethical committee of Universiti Sains Malaysia and research committee of Ministry of Science, Technology and Innovation (MOSTI).

\section{Patient selection}

All patients of locally advanced cervical cancer patients with bulky FIGO stage IB ( $>5 \mathrm{cms}$ ), IIA, IIB, IIIA, IIIB and IVA were recruited for the study. Patients with age range of 20 to 80 years, histopathological proof of cancer, willing to voluntarily participate and sign informed consent was allowed to enter in this trial. However patients with past history of radiotherapy and chemotherapy, connective vascular disease and uncontrolled diabetes mellitus were excluded from the study. A thorough clinical evaluation and pelvic examination under anesthesia (EUA) was performed to assess local primary disease and primary tumor volume. Contrast enhanced CT scans was performed to assess gross tumor volume and extent of disease. Routine full blood count, liver function test, chest radiograph were obtained before starting treatment. Histopathological evaluation of the biopsy specimen was mandatory, and cystoscopy and proctosigmoidoscopy was advised in advanced cases.

\section{Treatment protocol}

In this study, patients were simulated using conventional simulator (Mevasim, Siemens) and check films were obtained for verification and record. The upper border of the antero-posterior field was kept at L4/ L5 junction and lower border at 2-4 cms below the palpable tumor in the vagina during simulation. The lateral border included $2 \mathrm{cms}$ lateral to the lateral pelvic brim. In patients with antero-posterior separation more than $20 \mathrm{cms}$ were treated with 4 -field box-technique. The upper and lower border remained same as antero-posterior field, however the posterior border bisect the S2 sacral vertebra and anterior border placed behind the symphysis pubis. The dose was calculated at the midplane in antero-posterior technique and at the isocenter in 4-field box technique. A tumor dose of $42 \mathrm{~Gy}$ in 30 fractions (140cGy per fraction) was delivered over 10-days using a 6-MV linear accelerator (Primus, Siemens). Each fraction was delivered 3-times a day at an interfraction interval of 6 hours (i.e. 800 hours; 1400 hours and 2000 hours) continuously without weekend break. We did not apply any mid-line shield during external radiotherapy treatment.

One to two week following completion of external beam radiotherapy, two fractions of HDR intracavitary brachytherapy was delivered using microSelectron HDR unit (Nucletron BV, The Netherlands) at weekly interval. The brachytherapy applicators were inserted under general or spinal anesthesia. Individual computer treatment planning was done using Plato treatment planning system (Nucletron BV, The Netherlands). The calculation was based on the recommendations of ICRU-38 guidelines [11]. A HDR radiation dose of $9 \mathrm{~Gy}$ was prescribed to the Manchester point-A. The rectal and bladder dose was restricted to $60 \%$ and $80 \%$ of the Manchester point-A dose. The dose optimization was performed utilizing dwell time optimization technique. The patients with suboptimal regression and deformed geometry were treated with boost external radiotherapy dose of $20 \mathrm{~Gy}$ in 10 fractions over 2-week period.

\section{Monitoring}

The radiation side-effects of the pelvic sensitive end-organs were assessed using radiotherapy and oncology group (RTOG) grading system. Patients were monitored daily for recording of acute toxicity and on subsequent follow up for late toxicity. Each and every adverse event was recorded systematically in the case report form. All patients were hospitalized to observe any side-effects and change in body weight every week. A validated World Health Organization (WHO) Quality of life in Bahasa Malaysia language was recorded for every patient before and after the completion of external beam radiotherapy and before brachytherapy. The performance status was recorded using ECOG performance scale.

Clinical examination and examination under anesthesia (EUA) was done in all cases after completion of radiotherapy, and clinical pelvic examination alone on subsequent follow up visits in combined multidisciplinary gynecologic oncology clinic at 2-months interval. A CT scan was performed every 6-months interval to assess the abdominopelvic disease status. The results of clinical assessment were documented as complete response (CR), partial response (PR) or stable disease (SD) at the end of 6-week post radiotherapy. On follow up the disease status was evaluated as no evidence of disease (NED), local recurrence or metastatic disease.

\section{Observations}

\section{Patient characteristics}

The study was conducted between September 2003 to July 2007

\begin{tabular}{|l|l|}
\hline Response to radiotherapy & $12(48 \%)$ \\
\hline Complete response & $10(40 \%)$ \\
\hline Partial response & $03(12 \%)$ \\
\hline Stable disease & $12(48 \%)$ \\
\hline Disease status on last follow up & \multicolumn{2}{|l|}{} \\
\hline No evidence of disease & $08(32 \%)$ \\
\hline Local recurrence & $04(16 \%)$ \\
\hline Metastatic disease & $04(16 \%)$ \\
\hline Lost to follow up & \\
\hline Survival & $32-$ months (range 7-76months) \\
\hline Median follow up & 51 months \\
\hline Median Survival & $72 \%$ \\
\hline 24months survival & \\
\hline
\end{tabular}

Table 1: Outcome of treatment.

\begin{tabular}{|l|l|l|l|l|l|}
\hline \multicolumn{2}{|l}{ Toxicity } & Gotal (\%) & G2 & G3 & G4 \\
\hline Acute Morbidity & $04(16 \%)$ & 3 & 1 & 0 & 0 \\
\hline fever & $07(28 \%)$ & 5 & 2 & 0 & 0 \\
\hline Nausea & $06(24 \%)$ & 6 & 0 & 0 & 0 \\
\hline Vomiting & $16(64 \%)$ & 9 & 4 & 2 & 1 \\
\hline Fatigue & $07(28 \%)$ & 7 & 0 & 0 & 0 \\
\hline Abdominal pain & $13(52 \%)$ & 8 & 4 & 1 & 0 \\
\hline Diarrhoea & $02(8 \%)$ & 2 & 0 & 0 & 0 \\
\hline Tenesmus & & & & & \\
\hline Late Morbidity & $02(08 \%)$ & 0 & 2 & 0 & 0 \\
\hline Rectal bleeding & $01(4 \%)$ & 0 & 1 & 0 & 0 \\
\hline Subcutaneous fibrosis & $01(4 \%)$ & 0 & 0 & 0 & 0 \\
\hline Spinal pain & & & & \\
\hline
\end{tabular}

Table 2: Acute and late complications associated with CHART. 
Citation: Biswal BM, Ahmad NM, Hanafia ZA, Zakaria A, Othman NH, et al. (2011) Pilot Study on Continuous Hyperfractionated Accelerated Radiotherapy (CHART) and High Dose Rate Brachytherapy in Locally Advanced Cervical Cancer. J Cancer Sci Ther 3: 125-129. doi:10.4172/1948-5956.1000073

\section{Change of QoL (CHART)}

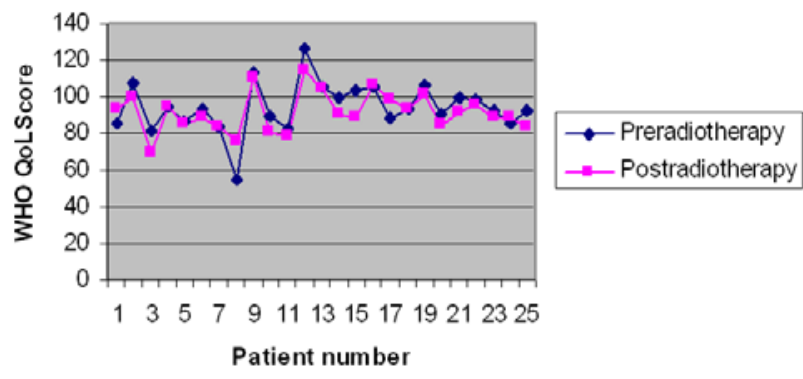

Figure 1: The change in WHO quality of life score before and after treatment.

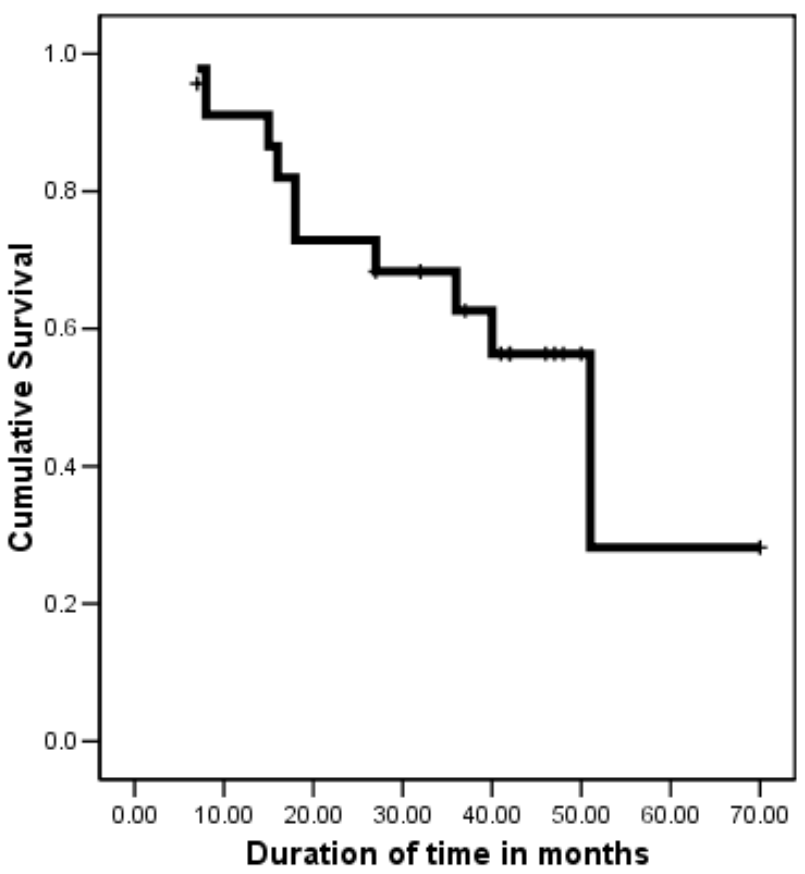

Figure 2: Kaplan-Meier Survival graph showing overall survival.

and 25 patients of invasive cervical cancers were recruited. The median age was 50-years (range 41-68 years). External beam radiotherapy was completed in all patients as per the protocol. The overall duration of external radiotherapy was 10 days. Out of 25 patients, 23 patients completed full courses of external radiotherapy and brachytherapy as per protocol as two patients were not suitable for brachytherapy insertion due to unfavorable pelvic geometry.

The immediate post radiotherapy response was assessed at the end 6-week of radiotherapy treatment. There were 12(48\%) complete response (CR) and 10(40\%) partial response (PR) and 3(8\%) stable disease observed at the end of 6-weeks. On follow-up, local recurrence was observed among $32 \%$ of patients, whereas metastatic disease was discovered in $16 \%$ patients (Table 1 ).

All patients were monitored daily for the development of acute complication and on subsequent follow-up for late complications. The most common acute morbidities were vomiting, nausea, diarrhea, fatigue and fever. The morbidities were grade- 1 and 2 and described in Table 2. The late morbidities were very few noted among 4-patients distributed as rectal bleeding (2), subcutaneous fibrosis (1), and spinal pain (1). The quality of life was assessed using WHO general quality of life scale performed before and after treatment which did not show any difference and presented as Figure 1.The median follow up period was 32-months with a. median survival on Kaplan-Meier survival analysis was 51 -months (Figure 2). The 24 -month overall survival was $72 \%$. At the time of reporting 9-patients (36\%) have died of disease and one of the deaths was unrelated to cancer.

\section{Discussion}

Current study revealed encouraging response rate and acceptable grade1-2 acute toxicity among treated patients. The grade1-2 late toxicity was $16 \%$ at a median follow up duration of 32 -month. The dose fractionations used in our trial being $42 \mathrm{~Gy}$ in 30 fractions over 10 days. The biological equivalence in term of $\mathrm{BED}^{10}$ was $48 \mathrm{~Gy}$. The teletherapy dose was reduced to $42 \mathrm{~Gy}$ in our study as the patients were subsequently treated with HDR brachytherapy. The combined BED of external and intracavitary brachytherapy being 60Gy. The overall $(\mathrm{CR}+\mathrm{PR})$ response rate was $88 \%$ and the 24 -month survival rate was $72 \%$. Though we had incomplete treatment among 2-patients, however, the survival and local control and tolerance to treatment were acceptable. Further escalation dose per fraction to1.5 Gy t.i.d in 30 fractions over 10 days could improve outcome in term of response rate. Perhaps inclusion of concurrent chemotherapy to above CHART protocol might improve the outcome further by controlling distant metastases. However large number of patients with long term follow up is necessary for the validation of CHART in cancer cervix. The use of altered fractionation in cervical cancer is observed in various clinical trials as summarized in Table 3. However these studies suffered from limited sample size and higher late end-organ toxicity.

Treating three times a day continuously without week-end break is a novel fractionation scheme being developed by Saunders and Hoskins from Mount Vernon Hospital and Grays Laboratories London to treat advanced head and neck cancer. Reducing dose per fraction, total duration of treatment and total dose has resulted in better survival rate compared to conventional fractionated radiotherapy in head and neck cancers [7]. Subsequently trials on non-small cell lung cancer too demonstrated better local control rate without increased late toxicities [20,21]. A trial on advanced rectal cancers treated with similar CHART schedule (i.e. $54 \mathrm{~Gy}$ in 36 fractions over 12 days) was observed to be well tolerated and achieved optimal palliation [10]. The late complications were very minimal. Another published trial using 25Gy in 15 fractions over 5-days in CHART has been tried among T3 resectable rectal cancers as preoperative regimen. In this study, one patient developed grade- 3 bowel toxicity and 2 patient's grade- 2 toxicity out of 20 rectal cancer patients studied [22]. In another study by Henk et al CHART protocol was administered concurrently with radiosensitizer nimorazole in a phase-II study on head and neck cancers. 61 inoperable head and neck cancers were treated with 56.5Gy in 36 fractions over 12 days along with radiosensitizer nimorazole. The locoregional control of patients was $55 \%$ at 2 years suggesting positive effects of nimorazole [23]. Thus nimorazole supplementation could be an option in addition to CHART in cancer cervix in future. The mature data of CHART on late reacting tissues and response to treatment was consistent in the recently reported results of Mount Vernon group.

Altered fractionation in the pelvic region is always considered to be toxic due to presence of rapidly renewing tissues in the bowel and bladder. There is a common notion for the development of diarrhea and tenesmus from the earlier experience of hyperfractionated radiotherapy 
Citation: Biswal BM, Ahmad NM, Hanafia ZA, Zakaria A, Othman NH, et al. (2011) Pilot Study on Continuous Hyperfractionated Accelerated Radiotherapy (CHART) and High Dose Rate Brachytherapy in Locally Advanced Cervical Cancer. J Cancer Sci Ther 3: 125-129. doi:10.4172/1948-5956.1000073

\begin{tabular}{|c|c|c|c|c|c|c|c|c|}
\hline Author & Number & Stage & Dose(Gy) & dpf & Frequency & Duration & Local control & Complications \\
\hline Singh[12] & 24 & III & 29 & 5.8 & qw & 28days & $87.50 \%$ & $100 \%$ \\
\hline Muckaden[13] & 62 & III & 39 & 3 & od & 17days & $59 \%$ & $27 \%$ \\
\hline Komaki[20] & 81 & IIIV & $24-48$ & 1.2 & bid & 24days & $80 \%$ & $6.30 \%$ \\
\hline Faria[16] & 23 & III & 72 & 1.2 & bid & 30days & $48 \%$ & $9 \%$ \\
\hline Viswanathan[14] & 30 & II-III & 60 & 1.2 & bid & 35days & $87 \%$ & $60 \%$ \\
\hline MacLeod[17] & 61 & II-IV & 57.5 & 1.25 & bid & 31days & $66 \%$ & $27 \%$ \\
\hline Chun[19] & 31 & II-III & 59 & $1.8-1.5$ & bid & 20days & $84 \%$ & $13 \%$ \\
\hline Grigsby[15] & 81 & II-III & 60 & 1.2 & bid & 33days & $55 \%$ & $12 \%$ \\
\hline Matsuura[21] & 7 & I-IV & 70.8 CCB & $1.2-1.6$ & bid & 35days & $86 \%$ & NA \\
\hline Ohno[22] & 120 & II-III & 50 & 1.5 & bid & 35days & $84 \%$ & $5 \%$ \\
\hline
\end{tabular}

Table 3: Studies using altered fractionation schedules for the treatment of cervical cancer Abbreviations: Stage-FIGO staging, Gy Gray, bid twice a day at an interval of 6-hours, Complications- maximum rectal complications, CCB concomitant boost using 3D conformal therapy, dpf dose per fraction, od daily, qw weekly.

of the urinary bladder and cancer cervix. The acute bowel toxicities in conventional fractionation is in a range of $10-20 \%$ whereas with hyperfractionation the figure rise to $40-50 \%$ and the grade $3-4$ toxicity are $12 \%$. In our study we observed $56 \%$ grade- $1-2$ acute bowel toxicity however the bladder toxicity was rare. In the Glynne-Jones series, the bowel toxicity was $33 \%$ and very low in Brooks study [10,22]. A pilot study conducted by Saunders et al in 1986 on advanced cancer cervix with 12 days CHART technique was tolerable; however the result was not published due to slow recruitment of patients [9].

The main drawback of our study is logistic for the delivery of three fractions per day continuously in weekends. However considering the radiotherapy dropouts in our set up justify the adoption of above technique to complete radiotherapy and brachytherapy in time in very short time. The late toxicity in our series with 32 -month follow up were tolerable, however long-term follow up could be of value to study late effects in future.

In conclusion, we observed 10-days CHART technique as a safe and well tolerated regimen for the treatment of locally advanced cervical cancer resulted in optimal local control rate and toxicity profile. Further study should focus on optimal sample size, higher dose per fraction, and increased fractionation for HDR brachytherapy to improve outcome. In low volume disease concurrent chemoradiotherapy, hyperthermia or incorporation of radiosensitizing agents could improve therapeutic ratio in future.

\section{Acknowledgement}

The study was supported by the Ministry of Science, Technology, and Innovation (MOSTI) long-term grant No 06-02-05-1037 PR0024/09-07. We would likes to thanks the Research \& Ethics committee, Research assistant Miss Norashikin, Mr Azman, Mrs. Siti Nur Azma, Mrs. Hasmat Zakaria for their contributions and advise.

\section{References}

1. Horiot JC, Pourquier S, Schraub J (1989) Current status of the management of cancer of the cervix in daily practice and in clinical research. In BrachytherapyII. Proceedings of the $5^{\text {th }}$ International SELECTRON users' meeting 1988 , The Hague the Netherlands. RF Moulds (Ed) Nucletron International BV. The Netherland, 199-214.

2. Patel FD, Rai B, Mallick I, Sharma SC (2005) High-dose-rate brachytherapy in uterine cervical carcinoma. Int J Radiat Oncol Biol Phys 62: 125-130.

3. Rose PG, Ali S, Watkins E, Thigpen JT, Deppe G, et al. (2007) Long-Term Follow-Up of a Randomized Trial Comparing Concurrent Single Agent Cisplatin, Cisplatin-Based Combination Chemotherapy, or Hydroxyurea During Pelvic Irradiation for Locally Advanced Cervical Cancer: A Gynecologic Oncology Group Study. J Clin Oncol 25: 2804-2810.

4. Eifel PJ (2006) Concurrent chemotherapy and radiotherapy as the standard of care for cervical cancer. Nat Clin Pract Oncol 3: 248-255

5. Datta NR, Agrawal S (2006) Does the evidence support the use of concurrent chemoradiotherapy as a standard in the management of locally advanced cancer of the cervix, especially in developing countries. Clin Oncol 18: 306-312.

6. McArdle O, Kigula-Magambe J (2007) Contraindications to cisplatinum based chemotherapy radiotherapy in the treatment of cervical cancer in sub-Saharan Africa: Kampala Uganda. Radiother Oncol 83: 94-96.

7. Saunders MI (1994) Accelerated fractionation and its application in clinical radiotherapy. In Current Radiation Oncology (Tobias JS and Thomas PRM eds) Edwards Arnold, London.

8. Girinsky T, Ray A, Roche B, Haie C, Gerbaulet A, et al. (1993) Overall treatment time in advanced cervical carcinomas: a critical parameter in treatment outcome. Int J Radiat Oncol Biol Phys 27: 1051-1056.

9. Saunders MI (1999) Head and neck cancer: Altered fractionation Schedules The Oncologist 4: 11-16.

10. Saunders MI, Dische S, Barrett A, Harvey A, Griffiths G, et al. (1999) Continuous, hyperfractionated accelerated radiotherapy (CHART) in non-smal cell lung cancer: mature data from the randomized multi centre trial. Radiother Oncol 52: 137-148.

11. Glynne-Jones R, Saunders MI, Hoskins P, Phillips H (1999) A pilot study of continuous hyperfactionated accelerated radiotherapy in recta adenocarcinoma. Clin Oncol 11: 334-339.

12. International Commission on Radiation Units and Measurements (1985): Dose and volume specification for recording intracavitary therapy in gynecology. ICRU report 38, Washington, ICRU.

13. Singh K (1978) Two regimens with same TDF but differing morbidity used in the treatment of stage III carcinoma of the cervix. Br J Radiol 51: 357-362.

14. Muckaden MA, Budrukkar AN, Tongaonkar HB, Dinshaw KA (2002) Hypofractionated radiotherapy in carcinoma cervix IIIB. Tata memorial Hospital experience. Ind J Cancer 39: 127-134.

15. Viswanathan FR, Varghese C, Peedicayil A, Lakshmanan J, Narayan VP, et al. (1999) Hyperfractionation in carcinoma cervix: tumor control and late bowel complications. Int J Radiat Oncol Biol Phys 45: 653-656.

16. Grigsby P, Winter K, Komaki R, Marcial V, Eifel P, et al. (2002) Long-term follow-up of RTOG 88-05: twice-daily external irradiation with brachytherapy for carcinoma of the cervix. Int J Radiat Oncol Biol Phys 54: 51-57.

17. Faria DL, Ferrigno R (1997) Hyperfractionated external radiation therapy in stage IIIB carcinoma of the cervix: a prospective pilot study. Int J Radiat Onco Biol Phys 38: 137-142.

18. Macleod C, Bernshaw D, Leung S, Narayan K, Firth I et al. (1999) Accelerated hyperfractionated radiotherapy for locally advanced cervix cancer. Int J Radiat Oncol Biol Phys 44: 519-524.

19. Ohno T, Nakano T, Kato S, Koo CC, Chansilpa Y, et al. (2008) Accelerated radiotherapy for cervical cancer: Multi-Institutional prospective study of forum for nuclear cooperation in Asia among eight Asian countries. Int J Radiat Onco Biol Phys 70: 1522-1529.

20. Chun M, Kang S, Ryu H, Chang K, Oh Y, et al. (2000) Modified partial hyperfractionation in radiotherapy for bulky uterine cervical cancer: reduction of overall treatment time. Int J Radiat Oncol Biol Phys 47: 973-977.

21. Komaki R, Pajak TF, Marcial VA, Rotman M, Grigsby PW, et al. (1994) Twice daily fractionation of external irradiation without brachytherapy in bulky cance of the cervix. Phase I/II study of the Radiation Therapy Oncology Group 88-05. Cancer 73: 2619-2625. 
Citation: Biswal BM, Ahmad NM, Hanafia ZA, Zakaria A, Othman NH, et al. (2011) Pilot Study on Continuous Hyperfractionated Accelerated Radiotherapy (CHART) and High Dose Rate Brachytherapy in Locally Advanced Cervical Cancer. J Cancer Sci Ther 3: 125-129. doi:10.4172/1948-5956.1000073

22. Matsuura K, Tanimoto H, Fujita K, Hashimoto Y, Murakami Y, et al. (2007) Early clinical outcomes of $3 \mathrm{D}$ conformal radiotherapy using accelerated hyperfractionation without intracavitary brachytherapy in cervical cancer. Gynecol Oncol 104:11-14.

23. Hatton MQ, Martin JE (2010) Continuous hyperfractionated accelerated radiotherapy (CHART) and non-conventionally fractionated radiotherapy in the treatment of non-small cell lung cancer: a review and consideration of future directions. Clin Oncol (R Coll Radiol) 22: 356-364.

24. Sause W, Kolesar P, Taylor S, Johnson D, Livingston R, et al. (2000) Final results of phase-III trial in regionally advanced unresectable non-small cell lung cancer. Chest I 117: 358-364.
25. Brooks S, Glynne-Jones R, Novell R, Harrison M, Brown K, et al. (2006) Shortcourse continuous hyperfractionated accelerated radiation therapy (CHART) as preoperative treatment for rectal cancer. Acta Oncol 45: 1079-1085.

26. Hank JM, Bishop K, Shepherd SF (2003) Treatment of head and neck cancer with CHART and nimorazole: phase-II study. Radiother Oncol 66: 65-70.

27. Saunders MI, Rojas AM, Parmar MK, Dische S, Chart Trial Collaborators (2010) Mature results of a randomized trial of accelerated hyperfractionated versus conventional radiotherapy in head-and-neck cancer. Int J Radiat Oncol Biol Phys 77: 3-8. 